\title{
IOT Based Anti-Poaching Sensor System for Trees in Forest
}

\author{
Parthiban M, Dharani M, Kathiga S, Keruthika M
}

\begin{abstract}
Presently a-days there are numerous episodes about carrying of trees like Sandal, Sagwan and so forth. Poaching of monetarily valuable trees has turned into a noteworthy dangerous to the estate of these trees, making an ecological irregularity and hazard the common assets. Distinctive activities have been taken world broadly including foundation of International Anti-Poaching establishment (IAPF). To confine their pirating and to spare woodlands around the world some preventive estimates should be made. We have built up a framework which can be utilized to confine smuggling. This task shows a framework for observing woodland and its region depends on IoT based remote sensor organize innovation. This paper proposes a microcontroller essentially based enemy of poaching framework utilizing WSN innovation. The fundamental thought displayed in this paper include utilizing a cutting edge and a modern innovation in which poachers will be deserted and being gotten effectively there by disposing of Poaching exercises. The framework propose three unique activities of reactions, right off the bat: passages constantly get information about trees area utilizing sensor. The second activity can be called perception where by various picture preparing methods of the got pictures encompassing a trees and in this manner give a sufficient help with understanding what makes sudden development of the trees. The last activity is to send messages to the officer's PDAs about the trees and the area through sensor. Backwoods authorities are told when any occasion happens so fitting move can be made. Remote sensor organize innovation can help build up a vitality effective framework for checking the poaching of trees.
\end{abstract}

Keywords: Antipoaching, Arduino-Uno, Temperature Sensor, Tilt Sensor, Sound Sensor.

\section{INTRODUCTION:}

We have planned a framework which can be utilized to keep away from the sneaking of the trees which would thus stop the deforestation and maintain the Environmental solidness, which would solve one of the issues with the Global Warming. Poaching isn't identified with India just, China, Australia and African nations are likewise battling with same issue. Indian sandalwood costs 12000 to 13000 INR for every $\mathrm{kg}$. Where as in universal market Red Sanders costs INR 10 crore for each ton. These are generally helpful in the therapeutic sciences just as beautifying agents. As a result of immense measure of cash associated with moving of such tree woods and bunches of occurrences are going on of cutting of tree and their smuggling. The Indian sandalwood tree has turned out to be uncommon as of late, trying to control its conceivable misfortune the Indian

Revised Manuscript Received on April 12, 2019.

Parthiban M, Department of Computer Science and Engineering, V.S.B. Engineering college,Karur-639111, TamilNadu, India. (parthim@mitindia.edu)

Dharani M, Department of Computer Science and Engineering, V.S.B. Engineering college,Karur-639111, TamilNadu, India.

Kathiga S, Department of Computer Science and Engineering, V.S.B Engineering college, Karur-639111, TamilNadu, India.

Keruthika M, Department of Computer Science and Engineering, V.S.B. Engineering college, Karur-639111, TamilNadu, India. government is attempting to restrain the exportation of sandalwood. The primary target of this undertaking is to build up a framework which can be utilized to limit sneaking of sandalwood trees. For an individual, greatest admissible buy limit isn't to surpass $3.8 \mathrm{~kg}$ according to Govt. On the off chance that the tree is now government controlled, at that point its evacuation is disallowed whether on private or sanctuary grounds until the tree is thirty years of age. Pirating of sandalwood has made financial and lawfulness issues in territories circumscribing in India.

It's difficult to envision a world without trees, yet as of now there are just 2 genuine woodlands left on planet earth. The unstoppable force of life is progressively enduring an onslaught nowadays, on the off chance that not by industrialists and worldwide partnerships, at that point by generally conventional individuals who appear to have lost all association with nature. Carrying/burglary of most imperative trees, for example, sandal wood in woodlands, represents a genuine risk to timberland assets, causes critical financial harm and at last has a significant crushing impact on the condition everywhere throughout the world. This paper propose a microcontroller based anti poaching framework utilizing WSN innovation, which is fit for identifying robbery by checking the vibrations created by the cutting of trees or branches. Vibration information gathered by different tests on wood and simulated. The plan framework utilizes three sensors tilt sensor (to identify the tendency of tree when its being cut), temperature sensor (to distinguish woodland fires), sound sensor (for powerful identification of illicit logging for example indeed, even the sounds produced while axing out the tree are likewise detected). Information created from these sensors is constantly checked with the guide of Blynk App. As for the sensors, their yield gadgets are initiated through relay switch. For tilt sensor and sound sensor a buzzer is actuated and for temperature sensor a water siphon is initiated. Created information is put away in Blynk Server over the Wi-Fi module.

Old redwoods in California are being disfigured, as poachers cutting tool off their burls to move the uncommon wood on the bootleg market. As the National Parks Service (NPS) expressed in a 2014 discharge, the end of the burls can fundamentally debilitate the tree, abandoning them powerless against winds and floods, just as creepy crawly pervasion in their uncovered heartwood. Also, the misfortune can successfully separate a tree's hundreds of years long life expectancy, as the burl keeps becoming even after a redwood falls. As NPS expresses: "A burl from a

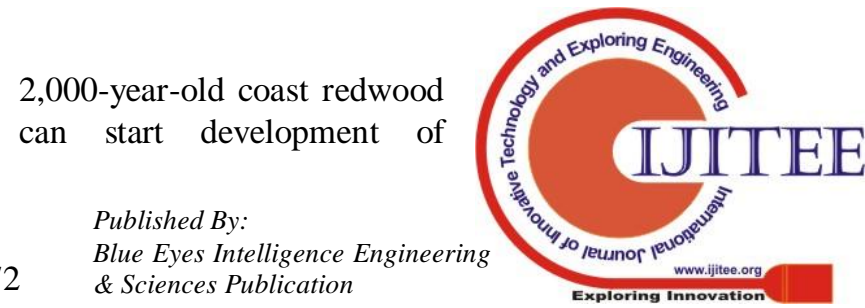


another tree that can live for an additional 2,000 years, in this manner the Latin name for coast redwoods, Sequoia simpervirens, which signifies 'everlastingly living.'" Tree poaching in the Redwood National Forest and other State Parks in the foggy area of Western United States between Northern California and Oregon is spreading, and park officers are finding that burlwood culprits are accomplishing progressively imperative components of mischief to these old trees, that are from time to time are over 2000 years old. Old advancement waterfront Redwood trees are one of earth's remaining basic fortunes, and after the outrageous logging of the latest couple of hundreds of years, relatively few remain. It's stunning by then to see that such an expansive number of trees are being attacked and denied of their burls, which are captivating improvements of wood that structure on the trunks of Redwoods, and confuse specialists. Burlwood is a baffling blend of bud tissue that conveys a remarkably superb dull red precedent, yet cutting into the burls from the trees can slaughter the tree or shield it from imitating.

Poachers are using evermore bold procedures, and park specialists trust this is the result of an economy in rot similarly as taking off rates of methamphetamine use in this region of the nation. 'Midnight burlers,' is the name they've been given by nearby individuals who are very exasperates about the rising example. In 2014, a tremendous, multi yearold Redwood was felled by midnight burlers just to cut a 500 -pound burl that was at least 60 or more feet up, addressing the main event when that an entire tree had been killed for a singular burl. A fiasco.

The burls bring a spotless advantage where they in the end up in the inventories of master who change the perfect burls into furniture and other fine things. Poaching burls from these incomprehensible trees surrenders them scarred and can render them logically defenseless to frightening little creatures and disorder, while moreover making it troublesome for the tree to emulate. Such pounding of nature for brief period advantage and money related benefit is a one progressively sign that mankind is in living in certified disharmony with nature. Poaching diminishes the dispersal of tree seeds in tropical woods by animals, which ominously impacts the air control limit of these forests. Without animals, an extensive number of these boondocks store less carbon over the long haul.

\section{RELATED WORK:}

Narhari R. Kotkar(sep 2004) at al - 200 teak trees cut, timber conveyed LUCKNOW. Imperiled red sandalwood seized from sprinters in Berhampur. The town China is home to a timber generation line that frames in excess of 5 billion pounds of wood for every year, by far most of which starts from Russia By conveying. Punjab Newsline Network on Saturday, 18 December 2010.The condition has gone all the more terrible as timber, worth lakhs and lakhs of rupees is essentially unfairly sold rightunder the nose of the concerned office. The Times of India, Ahmedabad. Plan to control between state conveying of woodlands woods.

Ghousia Sultana B, Jagadish R, Nadiya Noor Syed, Prof Nagashree C(may 2018) at al - Endangered red sandalwood seized from runners in Berhampur. The Times of India, Ahmadabad. Plan to control interstate carrying of backwoods woods. 3.200 teak trees cut, timber pirated in Lucknow. Punjab News line Network (eighteenth December 2010)- The circumstance has gone very more terrible as timber what's more, lakhs or Rupees are criminally being sold directly under the nose of division.

Prof. R. S. Mahajan (2017)at al - WSN: Remote Sensor Network is likewise called (WSAN) Wireless Sensor \&amp; amp; Actuator System. Fundamentally numerous applications can use WSN arrange with the end goal of WSN organize. We use here zig- bee module for transmitting \&amp; amp; accepting the data through WSN arrange. Also, likewise we use here various sorts of sensors to monitor the natural conditions, for example, temperature, vibration and human obstructions. WSN arrange use in numerous applications, for example, cataclysmic event aversion \&amp; amp; purchaser applications what's more, landside recognition to recognize the slight development of changes in different parameter. WSN organize is straightforwardness to use for worker of hub and every hub is associated with one sensor \&amp; amp; every sensor arrange hub has ordinarily a few sections.

Prasad R. Khandar, K. Deivanai (feb 2016) at al Smuggling and Illegal logging makes tremendous harm backwoods, financial aspects of maker nations and nearby networks. It's an intense and far reaching issue. In spite of the monetary significance of exchange timber and woodland items, the greater part of the universal nations have no lawful intend to stop or end such exercises on the grounds that in fact it's very difficult to recognize wrongfully carried timber also, different trees. Hence, some regulating acts against illicit exchanging of timber and different trees, is absent. Logical strategies to pinpoint the geographic starting point of timber are as of now being worked on. Conceivable activities should meet with WTO control of nondiscrimination to limit imports. They must be orchestrated in two-sided assent ions. Study led by UKIndonesian nations on illicit signing in 1998 recommended that around $40-45 \%$ of timber exchanging was illicit. This had caused more than $\$ 365$ million misfortune to nations. On the off chance that we incorporate and contrast and lawful collecting in addition trades propose that close about $88 \%$ is unlawful here and there. From Indonesia the vast majority of the unlawful wood generation is being conveyed out in Malaysia. This is key travel nation. While in Brazil, Amazon zone holds $80 \%$ unlawful exchanging this abuses government controls. At the center of unlawful logging is across the board debasement regularly called as 'Green Gold'. In Brazilian province of Para this issue is profoundly established. This examination was done by Greenpeace. As like timber, for mahogany there is no dependable legitimate path exists till date and key players in its exchange are still actuate in those areas.

LokhandeHarshali , KhalateVaishnavi

KapadekarSupriya , Kamble B.S(2017) at al First we look through the old papers we find that there are three units i.e., tree unit, sub server unit and the tree unit. In that units three

controllers, Zig- bee modules 
and MAX232 are utilized. So the framework turns out to be more convoluted and furthermore this framework contains the flex sensor. The disadvantage of utilizing such sensor is that it gives the exact perusing when it is twist. This disadvantage can be dodged by utilizing ADXL sensor which is 3- hub accelerometer. So we utilize this sensor and furthermore limit the circuit by lessening these three units into two units.

PratikshaBhuta

Ajay

Khandare

RakhshanAnjumShaikh(2017) at al - A remote sensor orchestrate involves various insignificant exertion, low power, and multi useful remote sensor centers, with recognizing, remote correspondences, and estimation capacities. These sensor center points pass on over short partition by methods for a remote medium and work together to accomplish a normal task, for example, condition checking, military perception, and mechanical system control.

The characteristics of the remote sensor frameworks are center point lifetime, versatility, steadfastness, gauge, control use, adaptability, and channel utilization, thusly it is critical to structure and develop a sensor organize which satisfies these genuine requirements and serves to increase the execution and the capability of the application mastermind. Because of the intricate idea of remote sensor orchestrate what's more, to draw out the lifetime of the sensors, profitable guiding counts must be used for data gathering.

Different leveled cluster based directing shows are considered as a champion among st the most capable guiding shows in remote sensor orchestrates due to its higher essentialness capability, arrange flexibility, and lower data re transmission. Different leveled designing is wound up being a convincing response for the issue of versatility and imperativeness efficiency. In a different leveled designing, the framework is secluded into different layers, and centers in particular layers perform various endeavors . Each slave center point reports to its individual expert center and passes on the distinguished credit to the pro. The expert center plays out the errand of data aggregation and sending the data further. The standard occupation of the expert center point is to send the distinguished data of the hostage to the sink for perception and checking.

There are particular topologies of different leveled coordinating for remote sensor organizes as referenced in. These topologies are disconnected in four classes, chainbased, tree-based, grid based, district based. Suggestions controlling strategies move as indicated by the application, each application hence needs a substitute technique for coordinating the information/hailing. Also, the essential objective of the arranged system should be to satisfy the characteristics of the remote sensor mastermind and the application requirements for which the structure is organized.

Ease is a clear low-control RF orchestrate show went for making little RF frameworks. Such frameworks normally contain battery worked devices which require long battery life, uninformed rate and low commitment cycle and have a foreordained number of centers talking clearly to each other. Correspondingly, we can structure a directing show for the organized system to screen the poaching of trees.

\section{PROPOSED SYSTEM:}

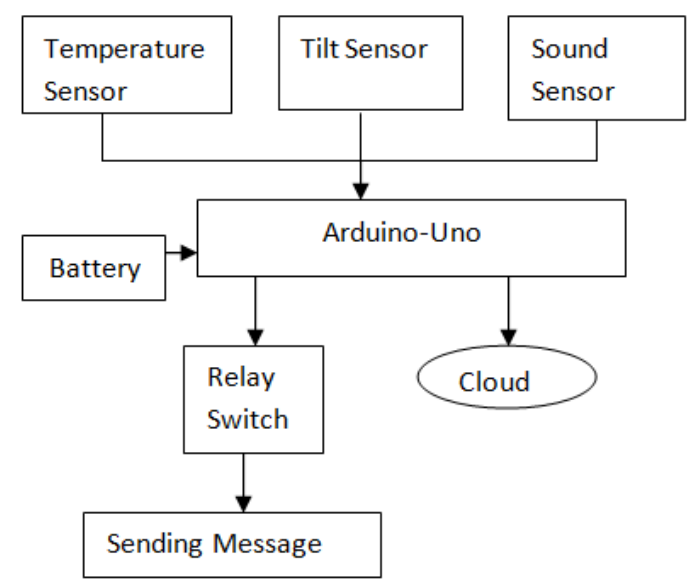

The plan framework utilizes three sensors:

1. TILT SENSOR: (to distinguish the tendency of tree when its being cut).

2. TEMPERATURE SENSOR :( to recognize timberland fires).

3. SOUND SENSOR :( for powerful recognition of illicit logging for example indeed, even the sounds produced while cutting out the tree is additionally detected).

Information produced from these sensors is consistently checked with the guide of Blynk App. As for the sensors, their yield gadgets are enacted through hand-off switch.

Arduino Uno is a microcontroller board subject to the ATmega328. It has 14 propelled data/yield, 6 straightforward information sources, a $16 \mathrm{MHz}$ aesthetic resonator, a USB affiliation, a power jack, an ICSP header, and a reset catch. It contains everything expected to help the microcontroller; just interface it to a PC with a USB connection or power it with an AC-to-DC connector or battery to start.

Tilt sensors are contraptions that produce an electrical banner that changes with an exact advancement. These sensors are used to check inclination and tilt inside a compelled extent of development. Every so often, the tilt sensors are alluded to as inclinometers in light of the way that the sensors essentially produce a banner anyway inclinometers make both readout and a banner. A tilt sensor is an instrument that is used for assessing the tilt in different tomahawks of a reference plane. Tilt sensors measure the tilting position with reference to gravity and are used in different applications.

They engage the basic area of presentation or propensity. Like mercury switches, they may moreover be known as tilt switches or moving ball sensors. The handiness of tilt sensors is influenced by factors, for instance, gravity, vibration, temperature, zero equalization, linearity, crosscenter point affectability, accelerating/deceleration, paralyze, clear detectable pathway between the customer and the intentional point, and arrangement of tilt sensors.

The key judgments of tilt sensors include:

- Number of tomahawks: The amount of tomahawks is a fundamental factor as it changes from application to 
application. In mechanical innovation, a twofold tomahawks tilt sensor is used. In PC diversion controllers and joysticks, a three tomahawks tilt sensor is required. In some propelled cell phones, four tomahawks sensors are used.

- Resolution: The base propensity recognized by the sensor.

- Sensitivity: The sensor's ability to react to little changes.

- Measuring range: The extent of propensity that can be managed by the sensor. A couple of sensors are fit for estimations of up to $10^{\circ}$ while others can cover an extent of up to $60^{\circ}$.

- Noise strength: Noise will by and large reason consonant bends in the capacity of the sensor, achieving yield assortment and an abatement of system efficiency. Maker's guidelines concerning uproar levels should be clung to.

- Vibration: Vibrations can bother the sensor's helpfulness, and from this time forward vibration block measures are required, especially when the sensors are used in severe conditions; for example in unpleasant landscape vehicles or building goals.

\section{TEMPERATURE SENSOR:}

It is a contraption which is arranged expressly to measure the hotness or crispness of a thing or in a space. Temperature sensor used in our undertaking is LM35.It's is a precision IC temperature sensor with its yield comparing to the temperature (in ${ }^{\circ} \mathrm{C}$ ). With LM35, the temperature can be assessed more correctly than with a thermistor. The working temperature broaden is from $-55^{\circ} \mathrm{C}$ to $150^{\circ} \mathrm{C}$. The temperature sensor is used to check the temperature. The temperature sensor uses the converter to change over the temperature motivator to an electrical regard. To scrutinize the temperature precisely and control the temperature in the advanced applications the temperature sensor is used even more extensively. The estimation of the temperature sensor is about the hotness or coolness of an article. The working base of the sensors is the voltage that scrutinized over the diode. If the voltage grows, by then the temperature rises and there is a voltage drop between the transistor terminals of base and maker, they are recorded by the sensors. If the qualification in voltage is upgraded, the basic banner is made by the device and it is explicitly in respect to the temperature.

\section{SOUND SENSOR:}

The Sound Sensor is a board that joins a receiver and some preparing circuit. It gives a sound yield as well as a double sign of the nearness of sound and a simple portrayal of sound's plentifulness. Sensors that distinguish sound or "hear" are just receivers. Electrostatic mouthpieces are oftentimes utilized for estimation since they are effectively scaled back, have level recurrence reactions over a wide recurrence run, and give notably high solidness. In examination, the piezoelectric receiver is broadly utilized basically as an amplifier for low-recurrence sound-level meters. Ultrasound is frequently utilized in range finding generally alluded to as SONAR (sound route and extending). Like RADAR (radio identification and extending), a ultrasonic heartbeat is produced in a particular course. In the event that an item is in the way of the beat, part or the majority of the beat is reflected back to the transmitter as a reverberation. Estimating the distinction between heartbeat transmission and reverberation shows the separation of the article. Estimation is influenced by saltiness and temperature when estimating SONAR beats in water and is utilized when separations are short and the exactness of the separation estimation is wanted to be more prominent.

\section{RELAY SWITCH:}

Hand-off Switch High voltage electronic devices can be controlled using exchanges. A Relay is a switch which is electrically worked by an electromagnet. The electromagnet gets instituted with a low voltage, for example 5 volts from a microcontroller and it dismantles a contact to speak to the choosing minute a high voltage circuit. A champion among the most good position is you can do with an Arduino is controlling higher voltage (120-240V) contraptions like fans, lights, radiators, and other nuclear family mechanical assemblies.

\section{BLYNK APPLICATION:}

Blynk was planned for the Internet of Things. It can control hardware remotely, show sensor data and can store data. It has 3 portions:

- Blynk App - It empowers us to make shocking interfaces for endeavors using diverse devices gave.

- Blynk Server - It is accountable for the impressive number of correspondences between the PDA and hardware.

- Blynk Libraries - for all the outstanding hardware stages - enables correspondence with the server and strategy all the drawing closer and out coming bearings.

\section{RESULT ANALYSIS:}

The site page called anti poaching dash board which shows the yield by utilizing the web. It accepts the contribution as region and gives status of that specific territory. The yield substance are Area name, all out tree ID's, Tree ID's which are fallen and Tree ID's which are alive. Likewise the dash board contains the invigorate button. On the off chance that somebody attempt to cut the trees the sensor sense the vibrations while produce cutting of trees the detected information will be send to the base station trough WI-FI the proprietor or security in charge for that specific territory will get the require the enrolled portable number which gives the data about the region, specific tree ID and furthermore gives the alert message to proprietor or security in control.

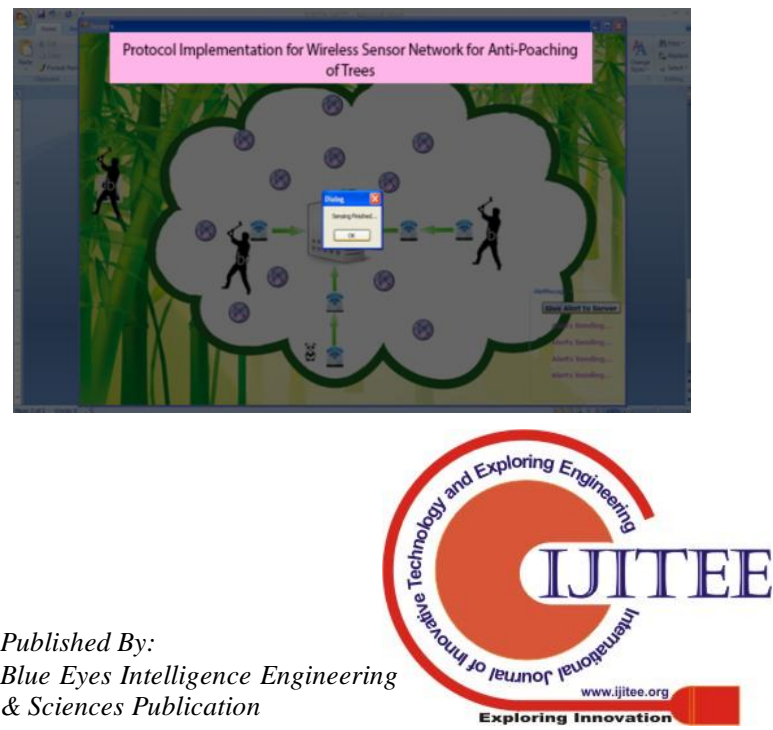




\section{CONCLUSION AND FUTURE WORK:}

This paper comprises of an ease and low power IOT based framework to identify the smuggling of trees. There are numerous approaches to secure trees, however here a brilliant technique for interfacing a few sensors around trees with a microcontroller was done. On the off chance that there are any unfortunate things happened in any area of the woodland. The sensor identifies and refreshes that data. The framework can be actualized utilizing Wireless Fidelity bolster which will be stretched out versatility up to $5 \mathrm{~km}$ range in the Forest Area. That is to imply the Forest experts about the tree's condition on $24 \times 7$ premise. This was conceivable in light of the fact that the installed unit has GPRS. Anyway the Tree's condition is under consistent observing in light of sensors. Thus it's an amalgamation of IoT, WSN and AWS to secure the Nature.

We have designed a system which can be used to avoid the smuggling of the trees. Future Scope Though the claim has been made that a Smart module has been developed to protect trees, future enhancements are required to make the system more rugged. - The Units / Hardware / Sensors have to be rugged. The Module should be placed in untraceable place on trees, not easily accessible to tree-destructors. Forest Authorities has to be suitably educated.

\section{REFERENCE:}

1. Akyildiz, I.F., Weilian S., Sankarasubramaniam, Y., Cayirci, E., "A survey on sensor networks," IEEE Communications Magazine, vol.40, no.8, pp.102-114, Aug. 2002.

2. Akyildiz, I.F., Weilian S., Sankarasubramaniam, Y., Cayirci, E., "Wireless Sensor Networks: A Survey," IEEE Computer, vol. 38, no. 4, pp.393-422, Mar. 2002.

3. Salehpour, A.-A.; Mirmobin, B.; Afzali-Kusha, A.; Mohammadi, S., "An energy efficient routing protocol for cluster-based wireless sensor networks using ant colony optimization," International Conference on Innovations in Information Technology, pp.455-459, 2008.

4. Hill, J. and Culler, D., "Mica: a wireless platform for deeply embedded networks," IEEE Micro, vol. 22, no. 6, pp.12-24, 2002.

5. Hill, J., Szewczyk, R., Woo, A., Hollar, S., Culler, D. and Pister, K., "System architecture directions for networked sensors," Proc. of ASPLOS, pp. 93-104, Nov. 2000.

6. Estrin, D., Govindan, R., Heidemann, J. S. and Kumar. S., "Next century challenges: Scalable coordination in sensor networks. Proc. of MOBICOM, pages 263-270, Aug. 1999.

7. Jun, Y., "The Characteristics and Energy Optimization Strategy of Wireless Sensor Networks," Journal of Chon Qing University (natural science edition), vol.25, no.5, pp.537-540, 2008.

8. Jie,C,; Benshun, Y., "A Low Power Consumption Multi-path Routing Optimization Protocols in Wireless Sensor Networks," Journal of Sensing Technology, vol.21, no.12, pp.2055-2060, 2008.

9. Jinging, Z.; Yongxi, H.; Yufei, C., "Wireless sensor network multi-path routing protocols," Journal of Computer Engineering and Design, vol.28, no.22. pp. 5417-5420, 2007.

10. Heinzelman, W.R.; Chandrakasan, A. and Balakrishnan, H "Energy-efficient communication protocol for wireless microsensor networks," Proceedings of the 33rd Annual Hawaii International Conference of System Sciences, vol. 2, pp. 3005-3014, 4-7 Jan 2000. 